\title{
Insular Cortex Activity Is Associated with Effects of Negative Expectation on Nociceptive Long-Term Habituation
}

\author{
Rea Rodriguez-Raecke, ${ }^{1 \star}$ Beril Doganci, ${ }^{2 \star}$ Markus Breimhorst,${ }^{2}$ Anne Stankewitz, ${ }^{1}$ Christian Büchel, ${ }^{1}$ Frank Birklein,${ }^{2}$ \\ and Arne May ${ }^{1}$ \\ ${ }^{1}$ Department of Systems Neuroscience, University Medical Center Hamburg-Eppendorf, D-20246 Hamburg, Germany, and ²Department of Neurology, \\ University Medical Center, 55101 Mainz, Germany
}

It is generally accepted that acute painful experience is influenced by context information shaping expectation and modulating attention, arousal, stress, and mood. However, little is known about the nature, duration, and extent of this effect, particularly regarding the negative expectation. We used a standardized longitudinal pain paradigm and painful heat test stimuli in healthy participants over a time course of 8 consecutive days, inducing nociceptive habituation over time. Thirty-eight healthy volunteers were randomly assigned to two different groups. One group received the information that the investigators expected the pain intensity to increase over time (context group). The other group was not given any information (control group). All participants rated the pain intensity of the daily standardized pain paradigm on a visual analog scale. In agreement with previous studies the pain ratings in the control group habituated over time. However, the context group reported no change of pain ratings over time. Functional imaging data showed a difference between the two groups in the right parietal operculum. These data suggest that a negative context not only has an effect on immediate pain but can modulate perception of pain in the future even without experience/conditioning. Neuronally, this process is mediated by the right opercular region.

\section{Introduction}

In recent years, a lot of effort has been put into understanding placebo analgesia, whereas relatively little research has focused on its negative equivalent, the nocebo effect. The nocebo effect refers to a worsening of symptoms when a subject relates the specific treatment to a negative outcome (such as that an inert substance or intervention will induce a hyperalgesic response) (Colloca and Benedetti, 2005). Whereas the effect of positive expectation is thought to be mediated by brain circuits typically involved in reward responses and motivated behavior (Enck et al., 2008), little is known regarding which brain areas integrate nociceptive input with negative contextual information and memory to provide cognitive exacerbation of pain affect. The few studies investigating nocebo effects using functional imaging described an involvement of the affective-cognitive pain pathway (medial pain system), namely the ACC, insula, operculum, prefrontal cortex, and hippocampus, and the authors administered inert treatment (sham acupuncture) in combination with a conditioning manipulation model that effectively made the participants believe that the treatment would enhance the pain experience.

Received April 29, 2010; revised July 9, 2010; accepted July 13, 2010.

This work was supported by grants from the Deutsche Forschungsgemeinschaft (MA 1862/2-3 and MA 1862/4-1 to A.M. and Bi 579/1-4), Rhineland-Palatinate Foundation, project 936, intramural grant of the University Medical Centre Mainz (to F.B.), and Neurolmage Nord. We thank all volunteers for the participation in this study and the Physics and Methods group at Neurolmage Nord in Hamburg and Falk Eippert for valuable discussions of the paper. ${ }^{*}$ R.R.-R. and B.D. contributed equally to this work.

Correspondence should be addressed to Dr. Arne May, Professor of Neurology, Department of Systems Neuroscience, University Medical Center Hamburg-Eppendorf, Martinistrasse 52, D-20246 Hamburg, Germany. E-mail: a.may@uke.uni-hamburg.de.

DOI:10.1523/JNEUROSCI.2197-10.2010

Copyright $\odot 2010$ the authors $\quad 0270-6474 / 10 / 3011363-06 \$ 15.00 / 0$
The authors reported that using this model, the subjective pain intensity ratings increased significantly more in the nocebo condition than in the control condition in which no expectancy/ conditioning manipulation was performed. Given that the functional imaging findings were primarily located in the affective-cognitive pain pathway, the authors concluded that their results underline the important role of anxiety in this process (Kong et al., 2008). This study was in part consistent with earlier studies (Sawamoto et al., 2000; Koyama et al., 2005; Keltner et al., 2006), namely that during nocebo hyperalgesia, brain activity in the ACC, insula, and operculum increases significantly during nocebo conditions. However, all of these studies investigated the immediate nocebo effect, i.e., effects that happen during the same session that the nocebo context (expectation of increased pain intensity) was given. This is highly relevant for the acute pain experience, but we know little regarding repetitive or longer pain experiences. How long into the future is the information that a doctor gives to his patient relevant, when the natural development of the condition is stable? We know that placebo and nocebo have immense immediate effects on pain perception but would expect that, if the given context is thwarted by the daily experience, the brain would recognize this prediction error and follow the natural outcome of repetitive input, i.e., habituate toward this stimulus. But is this true? Could negative expectation that is raised only once last for several days and counteract the natural course of perception? From a clinical point of view, two questions are important: How long is a negative context effective and how is its content processed, perceived, and modulated in the healthy human brain when negative expectations are raised toward repeated painful stimulation over several days? 
Habituation is generally defined as progressively smaller responses elicited by repeated stimuli (Glaser, 1966). Repetitive nociceptive stimulation in healthy subjects lessens the pain perception over time and causes habituation. This process is in part mediated by the antinociceptive system (Bingel et al., 2007; Teutsch et al., 2008).

The objective of the current study was to address how a negative manipulation that is only given once at the first day of an $8 \mathrm{~d}$ study phase can modulate the experience of pain immediately and in the following days of painful stimulation. Additionally, we were interested in which neural processes underlie these processes.

\section{Materials and Methods \\ Subjects}

A total of 44 healthy right-handed subjects ( 29 female) participated in the study. Four participants of the context group were excluded due to illness $(n=1)$, pregnancy $(n=1)$, technical problems $(n=1)$, and withdrawal from the experiment $(n=1)$. Two participants of the control group were excluded due to illness. This left complete datasets of 38 (25 female) participants. All participants were between 20 and 49 years old (mean age: context group $28.3 \mathrm{SD}=6.1$, control group $30.5 \mathrm{SD}=7.6$ ) and gave written informed consent acknowledging that (1) they would experience experimental pain stimuli, (2) all methods and procedures were clearly explained, and (3) they were free to withdraw from the experiment at any time. The study was conducted in accordance to the declaration of Helsinki and approved by the local Ethics Committee. All subjects had normal pain thresholds in the age- and gender-adjusted range and no history of any neurological or psychiatric disease. Particularly, none of the participants had any history of chronic pain, or acute pain up to 4 weeks before and during the study period.

\section{Pre-experimental phase and subjects' instruction}

A separate introductory session giving a detailed briefing about the experimental protocol was provided to all subjects $1-2 \mathrm{~d}$ before the actual experimental phase. During this session, all subjects made themselves familiar with the stimulation and rating procedures. Moreover, pain thresholds were determined using the method of limits (Engen, 1971; Fruhstorfer et al., 1976).

The participants were then randomly distributed into two different groups. All subjects were informed that the purpose of the current study was to investigate the behavior of the CNS in response to repetitive painful stimulation. The context group $(n=22)$ was additionally informed that repeated pain over several days will increase the pain sensation over time, e.g., from day to day. This information was given as a text sheet including a graph for visual illustration of the expected outcome before the heat pain stimulation series commenced. To guarantee that the essential instruction was perceived and understood, subjects read out the instruction to the examiner, and afterward, both examiner and subject signed this text. The second group (age and gender matched, $n=22$ ) served as a control group without any additional information.

\section{Study design}

The paradigm used was designed to achieve tolerable but effective nociceptive stimulation that could be repeated over several days without causing skin damage. For this purpose, a repetitive stimulation with a $48^{\circ} \mathrm{C}$ thermode-induced heat stimulus was applied, which inevitably activates peripheral heat nociceptors and evokes a moderate to intense painful sensation. The study phase consisted of an $8 \mathrm{~d}$ program of daily painful stimulation and has been used before by our group (Bingel et al., 2007, 2008; Teutsch et al., 2008). On each day, the subjects were exposed to one session of painful stimulation and pain ratings. Each stimulation session consisted of 10 blocks of heat stimuli with each block containing a series of six $48^{\circ} \mathrm{C}$ stimuli (each lasting $6 \mathrm{~s}$ ), resulting in a total number of 60 thermal stimuli. The interstimulus interval lasted $6 \mathrm{~s}$. Thermal stimuli were applied to the left volar forearm and delivered by a $30 \times 30 \mathrm{~mm}$ Peltier device (TSAII, Medoc). Five seconds after the sixth thermal stimulus of each pain block, the subject was prompted to rate the average sensation for the last six painful stimuli on a $0-100$ visual analog scale (VAS) with a button box. The VAS consisted of a bar moving from the left to the right end of the visual screen indicating sensations from 0 (no sensation) to 100 (maximum pain) The VAS was programmed using Presentation and presented on a visual computer screen during the training and the imaging sessions. To evaluate changes in pain processing and pain perception over time, functional MRI was performed on days 1 and 8 of the experimental phase, as well as $90 \mathrm{~d}$ after the stimulation epoch for $1 \mathrm{~d}$. Exactly the same protocol was used on all days, i.e., on each day of the program including the scanning days. Pain thresholds at the site of stimulus application were obtained analogous to the pre-experimental phase (methods of limits) on each scanning day before the scanning procedure. Skin conductance levels were measured in parallel to fMRI measurements on each scanning day during the experiment.

\section{Data acquisition}

Skin conductance level. Skin conductance levels (SCLs) were obtained using $\mathrm{Ag} / \mathrm{AgCl}$ electrodes (Red Dot monitoring electrode; 3M Health Care) attached to the hypothenar of the subjects' left hands. The same dermatome (C8) was chosen for both electrodes to control for possible recording differences between dermatomes. CED 2502 was used to amplify the skin conductance signal, a CED micro1401 mkII to digitize the signal at $100 \mathrm{~Hz}$, and Spike2 software to record and store the data (all equipment by Cambridge Electronic Design).

All data were resampled to $10 \mathrm{~Hz}$, smoothed with a $1 \mathrm{~s}$ Gaussian kernel (full width at half maximum), and $z$-transformed, and then an ANOVA with repeated measurement including time as a within-subject factor (days 1-8) and group as a between-subject factor (context vs control) was calculated. Amplitudes were determined as the mean in the analysis interval.

Image acquisition. The fMRI acquisition was performed on a 3 tesla Siemens TRIO MRI System with a 12-channel headcoil. For the functional scans, 42 axial slices with a thickness of $3 \times 3 \times 3 \mathrm{~mm}$ (no gap) were obtained using a gradient echo-planar (EPI) T2-sensitive sequence (TR $2.62 \mathrm{~s}$, TE $30 \mathrm{~ms}$, flip angle $80^{\circ}$, field of view $192 \times 192 \mathrm{~mm}$ ). Structural T1-weighted MRI-scans were acquired additionally for each subject by using a 3D-FLASH sequence (TR $15 \mathrm{~ms}$, TE $4.9 \mathrm{~ms}$, flip angle $25^{\circ}, 1 \mathrm{~mm}$ slices, field of view $256 \times 256 ; 240$ slices).

Image processing and statistical analysis ( $\mathrm{fMRI}$ ). Image preprocessing and statistical analysis were performed with SPM5 (www.fil.ion.ucl.ac. $\mathrm{uk} / \mathrm{spm}$ ) running under Matlab (MathWorks). Preprocessing involved realignment on the first EPI-volume, spatial normalization (Friston et al., 1994) to a standard EPI template (Evans et al., 1994), and smoothing with a $10 \mathrm{~mm}$ Gaussian kernel. For data analysis, the approach of the general linear model (GLM) was used, modeling the events "pain" and "motor response" (related to the VAS rating) and, moreover, the value of the VAS rating as a parametric regressor in a first-level matrix for each subject and session. The realignment parameters served as additional regressors (cutoff $3 \mathrm{~mm}$ translation and rotation). Coefficients for all regressors were estimated with the approach of least squares, and effects were tested with appropriate linear contrasts of the parameter estimates for the hemodynamic response function (HRF) regressor, resulting in a $t$ value for each voxel. The main effect of pain from the first-level analysis was used in a second level matrix to contrast groups and time intervals in a full factorial model. We were interested in effects of pain stimulation for both groups and differences between groups in all three sessions. The significance level for all contrasts was set as $p<0.001$ uncorrected. Group comparisons were performed for all time intervals and additionally for days 1 and 8 separately.

In an additional model, a finite impulse response (FIR) basis function was defined to display activation time courses. The poststimulus blood oxygen level-dependent (BOLD) response was averaged and a bin width of $2.62 \mathrm{~s}$ was used to model a total of 10 bins from 0 to $26 \mathrm{~s}$ after stimulus onset as regressors. This basis set uses each time bin individually to model the BOLD response at that time. Following a first-level analysis for each subject, all parameter estimates images were entered into a randomeffects analysis.

Behavioral data analysis. To analyze differences in pain intensity ratings and pain thresholds between days 1 and 8 with regard to both 

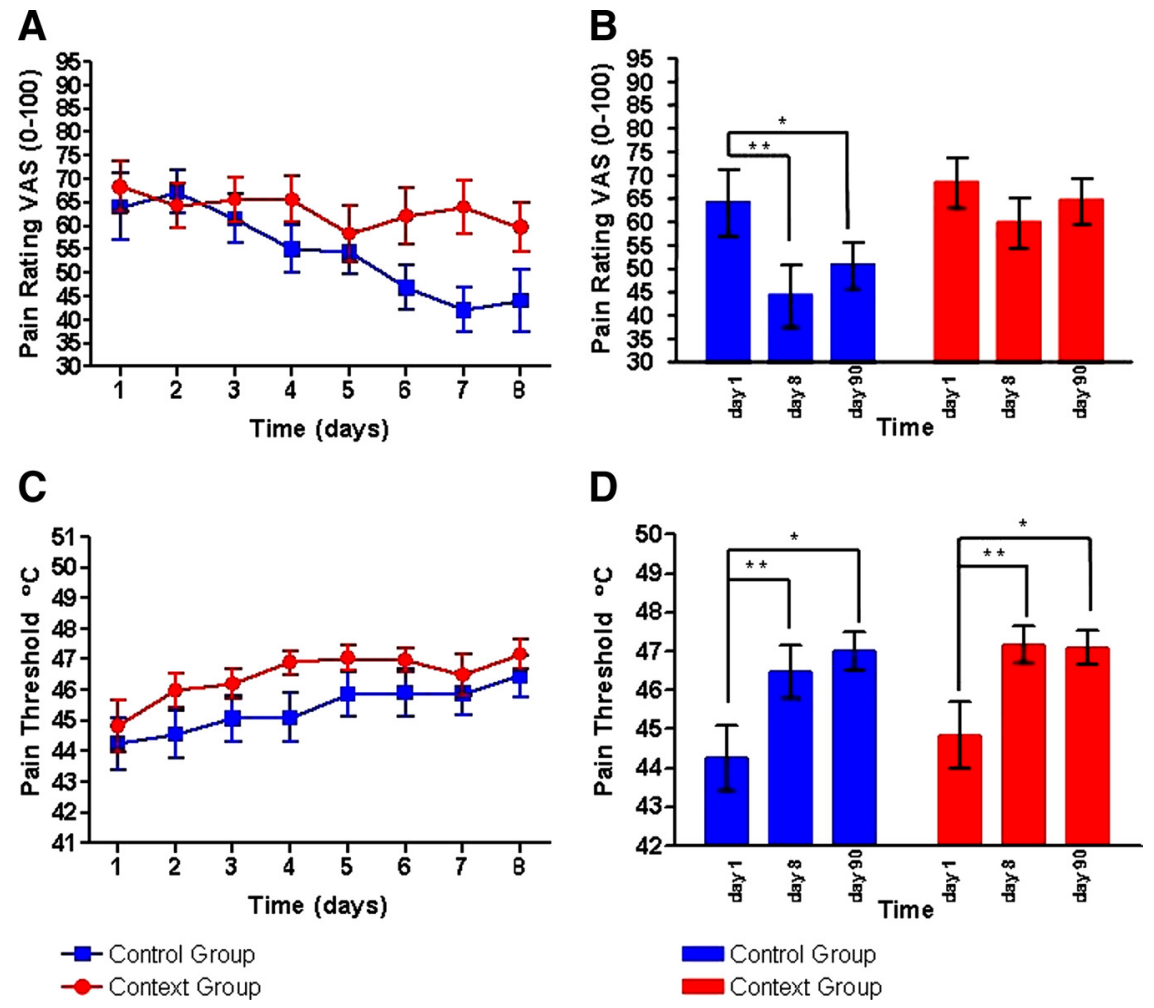

Figure 1. Pain ratings and pain thresholds for the context group (red) and the control group (blue). The upper row $(\boldsymbol{A}, \boldsymbol{B})$ displays the pain ratings on a visual analog scale from 0 (no pain) to 100 (worst pain imaginable) for the $8 \mathrm{~d}$ of the experiment $(\boldsymbol{A})$ and for the scanning days $(\boldsymbol{B})$. A significant day $\times$ context interaction was found between the context and the control group. The lower row $(\boldsymbol{C}, \boldsymbol{D})$ displays the pain thresholds for heat pain (when heat becomes painful) in degrees Celsius for the $8 \mathrm{~d}$ of the experiment $(\boldsymbol{C})$ and for the scanning days $(\boldsymbol{D})$.

groups, an ANOVA with repeated measurement including the withinsubject factor "day" (days 1-8) and the between-subject factor "context" (context vs control) was used. Normal distribution of pain ratings were examined by using Kolmogorov-Smirnov test, and homogeneity of error variances was checked using Levene test. Greenhouse-Geisser Epsilon was used to correct for possible violations of sphericity. Pairwise comparisons and simple effect tests were calculated by using Fisher's least significant difference (LSD). Statistical significance was considered as $p<0.05$. All statistical procedures were calculated using SPSS 15.0 for Windows.

\section{Results}

The pain thresholds for all subjects at the start of the study lay within the normal range of an age- and gender-matched sample of normative values, which are in accord with those provided by the manufacturer of the thermode (Wiech et al., 2005) as well as another study on normative data of various sensory tests, including heat pain thresholds from our own and cooperative laboratories (Rolke et al., 2006).

\section{Behavioral results}

Pain intensity ratings

Analysis of the pain ratings revealed a main effect of time on pain ratings $\left(F_{(8,296)}=5.18, p=0.001\right)$, i.e., a linear decrease of ratings from day 1 to day 6 ( $p=0.018)$, day 7 ( $p=0.010)$, and day 8 $(p=0.001)$. A significant time $\times$ context interaction revealed differences in the time course of pain ratings with respect to the context group $\left(F_{(8,296)}=2.67, p=0.043\right)$. Whereas the context group showed no differences in pain intensity ratings between day 1 and day $8(p=0.123)$ and between day 1 and day 90 ( $p=$ $0.529)$, the control group showed a significant reduction in pain ratings between day 1 and day 8 ( $p=$ $0.001)$ and day $90(p=0.032)$. In this group, the difference to day 1 was also significant for day $6(p=0.013)$ and day 7 ( $p=0.003$ ). Within session, both groups showed a sensitization. We conducted a $t$ test, which showed that the groups did not differ significantly within session.

\section{Pain thresholds}

Pain thresholds were influenced by time $\left(F_{(8,296)}=11.54, p<0.001\right)$ in both groups indicated by a significant increase of thresholds from day 1 to day 3 ( $p=0.008)$, day 4 $(p=0.002)$, day $5(p<0.001)$, day $6(p<$ $0.001)$, day $7(p=0.002)$, day $8(p<$ $0.001)$, and day $90(p<0.001)$. No significant interaction with context was observed $\left(F_{(8,296)}=1.05, p=0.401\right)$ pointing at a similar increase of pain threshold over time from day 1 to day 90 (Fig. 1).

\section{Skin conductance levels}

During each MR session, skin conductance level measurements were performed to detect the autonomic arousal level in response to painful stimulation. Due to technical problems, SCL-Data of 11 volunteers (context group: $n=3$, controls: $n=8$ ) could not be obtained. On day 1 , there was no difference of skin conductance level between groups. On day 8 , the skin conductance level showed a trend to increase in the context group compared to the control group. This difference did not reach significance due to the extent of noise in the data, namely a higher in-group variance than between groups. On day 90, there was again no difference between groups.

\section{Functional imaging results}

\section{Effect of condition and effect of time}

The group analysis for the repetitive painful thermal stimulation showed a significant activation in brain regions associated with pain processing (Treede, 1999; Peyron et al., 2000; Schnitzler and Ploner, 2000; Apkarian et al., 2005) in both groups for all three days $[p<0.001$, uncorrected but also reaching $p<0.001$ familywise error (FWE) corrected]. These areas comprise insular cortex, midcingulate cortex, parietal operculum, dorsolateral prefrontal cortex (DLPFC), secondary somatosensory cortex (SII), cerebellum, and brainstem. For $t$ values and $p$ values for specific regions, see Table 1 . This finding was observed for all imaging sessions (day 1, 8, and 90).

\section{Effect between groups}

We tested for differences of activation following painful stimulation between the two groups independent of the time intervals. Contrasting the main effect of pain stimulation between groups showed a significant increase of activation of the right operculum $\left([x=51 ; y=6 ; z=3] ; T_{(1,110)}=3.18 ; p<\right.$ 0.001 uncorrected) (Fig. 2) in the context group compared to the control group. Lowering the threshold to $p<0.05$ uncorrected revealed the opercular activation to be bilaterally. To illustrate the time course of the BOLD signal in the peak voxel 
of opercular activation during the pain condition, the averaged poststimulus response resulting from FIR basis set analysis is plotted in Figure 2.

Additionally, we performed a analysis for the group comparison on day 1 to see whether differences between groups are already evident on day 1 and found no significant differences between groups ( $p<0.001$ uncorrected). Focusing on regions known to be involved in negative expectation and anxiety, such as the amygdala, we used a standard mask specified for the right amygdala (Amunts et al., 2005) and found that it survived smallvolume correction $(t=1.87$, peak voxel $x=24, y=-6, z=$ -12). Defining ROI spheres using the WFU PickAtlas v2.4A (http://fmri.wfubmc.edu/cms/software\#PickAtlas), we found additionally the bilateral insula $(t=2.68)$, bilateral thalamus $(t=$ $2.50)$, and bilateral superior frontal gyrus $(t=2.23)$ in the context group compared to the control group.

In the group contrast at day 8, the control group showed increased activation in the midbrain, compared to the context group ( $p<0.05$ uncorrected). The context group showed, relative to the control group, an increase in activation in the operculum, frontal cortex, and the pons $(p<0.05$ uncorrected).

\section{Discussion}

We combined functional imaging and an expectation manipulation model to investigate the neural substrates of negative expectation over $8 \mathrm{~d}$ using a standardized heat pain on the left forearm. This heat pain paradigm is known to induce a robust habituation to repetitive nociceptive stimulation over time (Bingel et al., 2007; Teutsch et al., 2008; Rennefeld et al., 2010). We observed that the control group (the group with no context-specific information) showed a significant reduction in pain ratings between day 1 and day 8 , i.e., the control group habituated as expected. The context group, who received the information that pain increases over time when applied repetitively, did not habituate and reported a constant and invariable pain experience over all eight days. Of note, the context information referred specifically to the pain experience over time, and no information was given regarding the first session. As can be expected, the pain ratings in both groups were identical from day 1 until day 5 , and only then diverged significantly on days 6 to 8: The control group habituated and the context group did not. The placebo effect may occur through both expectation and conditioning mechanisms (Colloca and Benedetti, 2005), and a correlation between the degree of expected treatment effect and the subsequent placebo-induced analgesia has been observed (Price et al., 1999). Importantly, we did not use learning or conditioning paradigms, i.e., the negative information was only once given verbally. Our finding that expectation alone can shape the outcome is in line with an earlier work by Colloca et al. (2008), who suggested that learning is not equally important in nocebo hyperalgesia compared to placebo analgesia.
Table 1. The group analysis for the repetitive painful thermal stimulation showed a significant activation in brain regions associated with pain processing in both groups $(p<0.001$, FWE corrected)

\begin{tabular}{lrrrrr}
\hline & \multicolumn{1}{l}{ MNI } & & & & \\
\cline { 2 - 3 } Anatomical location & $x$ & $y$ & $z$ & & Peak $t$ scores \\
\hline R superior temporal gyrus & 54 & 0 & 3 & $<0.001$ & 14.09 \\
R operculum & 39 & -18 & 18 & $<0.001$ & 12.73 \\
R insular cortex & 39 & 6 & 9 & $<0.001$ & 12.15 \\
L superior temporal gyrus & -60 & 3 & 0 & $<0.001$ & 11.10 \\
L insular cortex & -36 & 6 & 9 & $<0.001$ & 10.73 \\
L anterior insular cortex & -27 & 27 & 6 & $<0.001$ & 10.20 \\
R midcingulate cortex & 6 & 12 & 42 & $<0.001$ & 9.61 \\
L cerebellum (VIII) & -18 & -69 & -51 & $<0.001$ & 7.03 \\
R cerebellum (crus1) & 30 & -78 & -27 & $<0.001$ & 8.28 \\
R DLPFC & 39 & 48 & 30 & $<0.001$ & 6.95 \\
R brainstem & 18 & -36 & -51 & $<0.001$ & 6.79 \\
L inferior frontal gyrus & -27 & 30 & -15 & $<0.001$ & 6.71 \\
L nucleus caudatus & -6 & 6 & 3 & $<0.001$ & 8.48 \\
\hline
\end{tabular}

This finding was observed for all imaging sessions (days 1,8 , and 90 ). The changes are tabulated in terms of the brain region and the corresponding Brodmann's area (BA). The $x, y, z$ coordinates are according to the MNI atlas. Each location is the peak within a cluster (defined as the voxel with the highest $Z$ score).

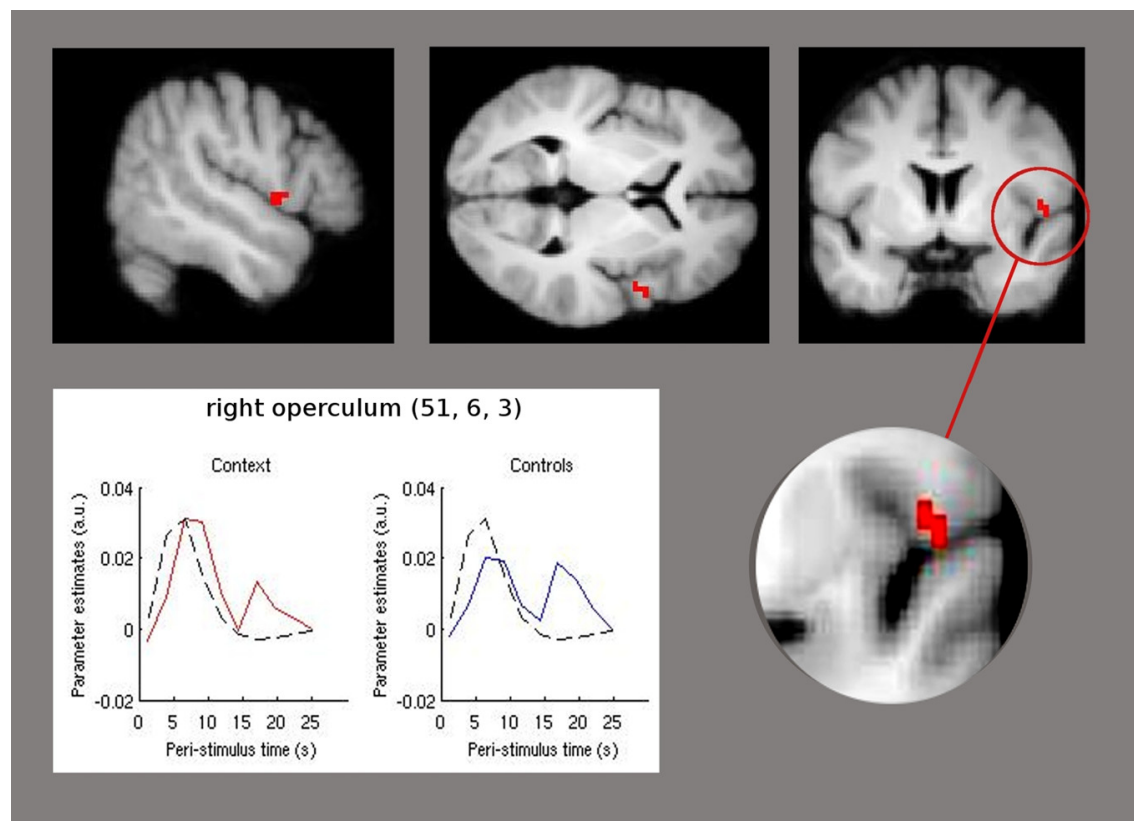

Figure 2. The context group showed significant higher activation ( $p<0.001$ uncorrected) of the right parietal operculum compared to the control group. Statistical parametric maps demonstrating this difference are superimposed in red on a structural $\mathrm{T}_{1}$-weighted MRI used for spatial normalization (upper row). Lower row, The plot is derived from an FIR basis set. Parameter estimates, diagramed on the $y$-axis, illustrate the averaged time course of the poststimulus BOLD signal in the peak voxel derived from the right parietal operculum at [51,6,3]. The red line represents the FIR of the context group, the blue line shows the FIR of the control group, and the black line presents the HRF. The $x$-axis indicates the poststimulus time in seconds from 0 to $26 \mathrm{~s}$, and the $y$-axis shows the parameter estimates that are directly proportional to the BOLD signal. The hemodynamic response of the right operculum shows an identical onset and duration of the finite impulse response for both groups, whereas the extent of activation following pain stimulation is higher in the context group.

Given that most groups found that the nocebo effect may be produced through a cortical network responsible for affective/ emotional and cognitive aspects of pain perception (Kong et al., 2008), we also obtained skin conductance levels of all participants on days 1,8 , and 90 to assess the arousal in response to painful stimulation. On days 1 and 90, there was no difference of skin conductance between groups. On day 8 , the skin conductance level increased in the context group compared to the control group. This difference did not reach significance due to the extent of noise in the data, namely a higher in group variance than 
between groups. Given that the finding was not significant, these data need to be seen with caution. However, the apparent difference between the control group and the context group only on day 8 (and, given the instruction, any change would have only then been suspected) suggests a higher anxiety level or perhaps anticipatory tension, contradicting a reporting bias. An even stronger argument against a reporting bias is the fact that the context group did not behave as instructed, i.e., the pain did not increase over time although the context explicitly suggested so. Thus, our data expand the knowledge of instruction effects on immediate pain experience and support the notion that a given context may modulate the perception of repetitive nociceptive input for at least $8 \mathrm{~d}$ into the future. This is clinically highly relevant as these findings suggest that a negative expectation raised verbally by a doctor only once in a clinical context may cause significant changes of the patient's perception in the future. It is also important to bear in mind that the patient's expectations of adverse effects at the outset of treatment may be pivotal regarding the occurrence of nonspecific side effects while taking active medication (Barsky et al., 2002).

Additionally, we show that this phenomenon is significantly associated with a selective activation of the parietal operculum. The parietal operculum has a substantial role in the cortical representation of pain (Treede et al., 2000; Fabri et al., 2002; Schlereth et al., 2003), which is further underlined by the fact that patients with lesions in the parietal operculum suffer from deficits in pain perception (Greenspan and Winfield, 1992). A cognitive-evaluative function of the parietal operculum is further confirmed by the parallel effects of a cognitive distracting task on the perceived intensity and the cerebral perfusion increase in the parietal operculum induced by the cold pressor test (Petrovic et al., 2000). Electrophysiological data in humans (Schlereth et al., 2003; Iannetti et al., 2005) and clinical observations in patients with cortical lesions (Greenspan et al., 1999) suggest the potential role of the parietal operculum as a nociceptive area that is activated very early after the onset of phasic noxious stimuli. More specifically, a recent functional imaging study by Kong et al. (2008) suggested that the nocebo hyperalgesia is associated with an activation of the bilateral insula, operculum, ACC, and left S1/M1 as well as the left hippocampus. In our study, the only difference in brain imaging data between the context group and the control group was in the right parietal operculum. Lowering the threshold to $p<0.05$ uncorrected, this finding is bilateral and additionally comprises the thalamus and right amygdala. The methodological differences between the study by Kong et al. (2008) and our study are manyfold, the most important one being that we investigated long-term rather than short-term intrasession effects and that the relative hyperalgesic effect in our study relied exclusively on the verbal information as opposed to conditioning.

Until now, there was sparse evidence whether long-term processes (e.g., habituation over days) may be distracted by a psychological intervention and what brain regions correspond to this. In our study, behavioral data and brain activation were significantly different between groups, depending on whether the negative context was introduced or not. What is even more important is that the instruction was only given once at the first day of the investigation, and that it was specifically designed to refer to a situation not in the present but some days in the future. Although the pain paradigm was kept strictly constant at all days, the negative expectation modulated, just as instructed, the perception of pain along the way and the pain experience between both groups became significantly different after nearly a week, on day 6 .
In all probability, placebo information that pain sensation is expected to decrease over time in this experiment would have led to an even stronger effect of habituation than the natural development (our unpublished data). Clinically, this information is highly relevant, given that clinical pain specialists are regularly asked about the natural course of a painful disease. Physicians have to bear in mind that uncareful nocebo information may have significant consequences at a much later time point. It has also implications for experimental pain studies, given that information presented at the beginning of a pain experience may modulate the outcome and processing of pain in the future. Simply telling the subjects that the aim of a given study is to understand mechanisms for chronification or reduction of pain could raise an expectation toward increased or reduced pain perception. Such instructions are usually not intentional and therefore not reported in the experimental protocol.

\section{References}

Amunts K, Kedo O, Kindler M, Pieperhoff P, Mohlberg H, Shah NJ, Habel U, Schneider F, Zilles K (2005) Cytoarchitectonic mapping of the human amygdala, hippocampal region and entorhinal cortex: intersubject variability and probability maps. Anat Embryol (Berl) 210:343-352.

Apkarian AV, Bushnell MC, Treede RD, Zubieta JK (2005) Human brain mechanisms of pain perception and regulation in health and disease. Eur J Pain 9:463-484.

Barsky AJ, Saintfort R, Rogers MP, Borus JF (2002) Nonspecific medication side effects and the nocebo phenomenon. JAMA 287:622-627.

Bingel U, Schoell E, Herken W, Büchel C, May A (2007) Habituation to painful stimulation involves the antinociceptive system. Pain 131:21-30.

Bingel U, Herken W, Teutsch S, May A (2008) Habituation to painful stimulation involves the antinociceptive system-a 1-year follow-up of 10 participants. Pain 140:393-394.

Colloca L, Benedetti F (2005) Placebos and painkillers: is mind as real as matter? Nat Rev Neurosci 6:545-552.

Colloca L, Sigaudo M, Benedetti F (2008) The role of learning in nocebo and placebo effects. Pain 136:211-218.

Enck P, Benedetti F, Schedlowski M (2008) New insights into the placebo and nocebo responses. Neuron 59:195-206.

Engen T (1971) Psychophysics I: discrimination and detection. In: Experimental psychology (Kling JW, Riggs LA, eds), pp 11-46. New York: Holt.

Evans AC, Kamber M, Collins DL, Macdonald D (1994) An MRI-based probabilistic atlas of neuroanatomy. In: Magnetic resonance scanning and epilepsy (Shorvon S, Fish D, Andermann F, Bydder GM, Stefan H, eds), pp 263-274. New York: Plenum.

Fabri M, Polonara G, Quattrini A, Salvolini U (2002) Mechanical noxious stimuli cause bilateral activation of parietal operculum in callosotomized subjects. Cereb Cortex 12:446-451.

Friston KJ, Holmes AP, Worsley KJ, Poline JP, Frith CD, Frackowiak RSJ (1994) Statistical parametric maps in functional imaging: a general linear approach. Hum Brain Mapp 2:189-210.

Fruhstorfer H, Lindblom U, Schmidt WC (1976) Method for quantitative estimation of thermal thresholds in patients. J Neurol Neurosurg Psychiatry 39:1071-1075.

Glaser EM (1966) The physiological basis of habituation. London: Oxford UP.

Greenspan JD, Winfield JA (1992) Reversible pain and tactile deficits associated with a cerebral tumor compressing the posterior insula and parietal operculum. Pain 50:29-39.

Greenspan JD, Lee RR, Lenz FA (1999) Pain sensitivity alterations as a function of lesion location in the parasylvian cortex. Pain 81:273-282.

Iannetti GD, Zambreanu L, Cruccu G, Tracey I (2005) Operculoinsular cortex encodes pain intensity at the earliest stages of cortical processing as indicated by amplitude of laser-evoked potentials in humans. Neuroscience 131:199-208.

Keltner JR, Furst A, Fan C, Redfern R, Inglis B, Fields HL (2006) Isolating the modulatory effect of expectation on pain transmission: a functional magnetic resonance imaging study. J Neurosci 26:4437-4443.

Kong J, Gollub RL, Polich G, Kirsch I, Laviolette P, Vangel M, Rosen B, Kaptchuk TJ (2008) A functional magnetic resonance imaging study on 
the neural mechanisms of hyperalgesic nocebo effect. J Neurosci 28:13354-13362.

Koyama T, McHaffie JG, Laurienti PJ, Coghill RC (2005) The subjective experience of pain: where expectations become reality. Proc Natl Acad Sci U S A 102:12950-12955.

Petrovic P, Petersson KM, Ghatan PH, Stone-Elander S, Ingvar M (2000) Pain-related cerebral activation is altered by a distracting cognitive task. Pain 85:19-30.

Peyron R, Laurent B, García-Larrea L (2000) Functional imaging of brain responses to pain. A review and meta-analysis (2000). Neurophysiol Clin 30:263-288.

Price DD, Milling LS, Kirsch I, Duff A, Montgomery GH, Nicholls SS (1999) An analysis of factors that contribute to the magnitude of placebo analgesia in an experimental paradigm. Pain 83:147-156.

Rennefeld C, Wiech K, Schoell ED, Lorenz J, Bingel U (2010) Habituation to pain: further support for a central component. Pain 148:503-508.

Rolke R, Magerl W, Campbell KA, Schalber C, Caspari S, Birklein F, Treede $\mathrm{RD}$ (2006) Quantitative sensory testing: a comprehensive protocol for clinical trials. Eur J Pain 10:77-88.

Sawamoto N, Honda M, Okada T, Hanakawa T, Kanda M, Fukuyama H,
Konishi J, Shibasaki H (2000) Expectation of pain enhances responses to nonpainful somatosensory stimulation in the anterior cingulate cortex and parietal operculum/posterior insula: an event-related functional magnetic resonance imaging study. J Neurosci 20:7438-7445.

Schlereth T, Baumgärtner U, Magerl W, Stoeter P, Treede RD (2003) Lefthemisphere dominance in early nociceptive processing in the human parasylvian cortex. Neuroimage 20:441-454.

Schnitzler A, Ploner M (2000) Neurophysiology and functional neuroanatomy of pain perception. J Clin Neurophysiol 17:592-603.

Teutsch S, Herken W, Bingel U, Schoell E, May A (2008) Changes in brain gray matter due to repetitive painful stimulation. Neuroimage 42 : 845-849.

Treede RD (1999) Transduction and transmission properties of primary nociceptive afferents. Ross Fiziol Zh Im I M Sechenova 85:205-211.

Treede RD, Apkarian AV, Bromm B, Greenspan JD, Lenz FA (2000) Cortical representation of pain: functional characterization of nociceptive areas near the lateral sulcus. Pain 87:113-119.

Wiech K, Seymour B, Kalisch R, Stephan KE, Koltzenburg M, Driver J, Dolan RJ (2005) Modulation of pain processing in hyperalgesia by cognitive demand. Neuroimage 27:59-69. 